\title{
REVIEW ON THE ROAD TRAFFIC NOISE ASSESSMENT
}

\author{
ALINA PETROVICI ${ }^{*}$, CLAUDIA TOMOZEI ${ }^{1}$, FLORIN NEDEFF $^{1}$, \\ OANA IRIMIA ${ }^{1}$, MIRELA PANAINTE-LEHADUS ${ }^{1}$ \\ "Vasile Alecsandri" University of Bacau, Faculty of Engineering, Department of \\ Mechanical and Environmental Engineering, Calea Marasesti, 157, Bacau, 600115, \\ Romania
}

\begin{abstract}
This paper presents a synthesis of current state of the assessment of road traffic noise in urban areas considering economic, social and legal aspects. Therefore, there were described several prediction methods of the urban traffic noise. These methods are useful in calculating the exposure of the population at noise levels which exceed the permissible limits. Mapping is one of the most common methods used for the assessment of noise. Whether it is industrial, airport, rail or road traffic noise, noise mapping provides accurate data needed later in developing action plans against noise. The road traffic noise assessments are performed periodically, and a representative picture of the noise in the analysed areas is obtained. Then, the action plans can be developed in order to reduce road traffic noise, where it is necessary.
\end{abstract}

Keywords: assessment method, noise map, traffic noise, noise prediction, noise legislation

\section{INTRODUCTION}

The transportation sector is one of the major contributors to noise in urban areas. Interrupted traffic flow noise is associated with the typical congested conditions, and has different characteristics from the noise generated by free flow on rural roads. These differences arise from various traffic flow characteristics such as heterogeneity of traffic, flow speeds etc., which is in contrast to traffic on rural roads, where the flow is generally continuous [1]. Generally, the noise effects are separated into two broad categories: auditory (noise-induced hearing loss) and non-auditory (behavioural and physiological effects) [2].

Strategic noise map is a tool for global assessment of exposure to noise from various sources in a given area or for overall predictions for the area. An increased interest on noise mapping was registered with the adoption of the European Environmental Noise Directive [3]. Noise maps had to be developed for all major agglomerations, major airports, roads and railways up to date [4]. The first stage of noise mapping was completed in 2007 when all member states of the European Union had to deliver noise maps for agglomerations and infrastructure specified in the legislation at that time. The second phase of noise mapping was planned until 2012 and covered a larger area than in 2007. At least every five years, strategic noise maps must be reviewed and if necessary revised [5].

Noise maps can be made with data obtained by calculations, based on known parameters such as: 3D terrain characterization, average running speed of vehicles and weather conditions etc. Most of the time, all necessary data for each of the examined areas is unavailable. Therefore, some assumptions and speculations have to be made to provide specific input to the calculation model. Because of this, whenever possible, the maps are "calibrated" by measurements taken at certain points [5].

\footnotetext{
* Corresponding author, email: alinapetrovici@ub.ro
}

(c) 2016 Alma Mater Publishing House 
Noise maps are developed for the following purposes [6]: investigate the noise conditions, focusing on identifying areas where maximum limits are exceeded; identify "hot spots" areas; inform the exposed population; assess the levels of noise at the sensitive facades of the buildings; for new land planning and construction in the noisy areas; investigate the noisy activities; compare the calculated and measured levels of noise; assess the economic losses caused by noise and cuts in property values (one $\mathrm{dB}$ increase in noise level corresponds to a decrease of $1 \%$ of the rental housing).

The main objective of the paper is to present some general aspects concerning the assessment of the urban road traffic noise taking into consideration several issues about the prediction methods and other ways of noise evaluation like noise measurements tested in different European countries and beyond.

\section{NOISE LEGISLATION}

The legislation aims to reduce exposure to environmental noise through harmonization of noise indicators and assessment methods by collecting information related to exposure to noise in the form of "noise maps" and making this information available to the public. On this basis, member states have developed action plans to solve the noise issues. Noise maps and action plans are reviewed at least at every five years [4].

The main purpose of the noise assessment is to define a common approach to avoid, prevent or reduce with priority the harmful effects, including annoyance, due to exposure to environmental noise. This requires a gradually implementation of the following actions [4]:

a) Evaluate the exposure to environmental noise, through noise mapping using evaluation methods;

b) Ensure that the information on environmental noise and its effects is available to the public;

c) Elaborate the action plans by the member states, based on the results of the noise maps for preventing and reducing environmental noise where necessary and particularly where exposure levels can be harmful for human health and preserve the quality of the environment in the quiet areas.

According to this directive, in developing the strategic noise maps, the $L_{d e n}$ and $L_{n i g h t}$ noise indicators are used; the $L_{d e n}$ indicator is the general noise level during the day, evening and night used to quantify the discomfort caused by exposure to noise and is calculated as in equation (1), and $L_{\text {night }}$ indicator is the sound level during the night and it quantifies sleep disturbances. The beginning of the day, evening, and night are determined by each Member State; the default hourly intervals are: 07.00 - 19.00, 19.00 - 23.00 and 23.00 - 07.00, local time [4].

$$
L_{\text {den }}=10 \lg \frac{1}{24}\left(12 \cdot 10^{\frac{L_{d a y}}{10}}+4 \cdot 10^{\frac{L_{\text {evening }}+s}{10}}+8 \cdot 10^{\frac{L_{\text {night }}+10}{10}}\right)
$$

World Health Organization (WHO) estimates that about $40 \%$ of the population is exposed to road traffic noise levels exceeding $55 \mathrm{~dB}(\mathrm{~A})$ and that over $30 \%$ of the same population is exposed to levels exceeding $55 \mathrm{~dB}(\mathrm{~A})$ overnight [7].

Directive 70/157/EEC and its amendments regarding noise on vehicles concerns the outside noise requirements of the vehicle while driving under test, it describes the test procedure and sets the noise limits. It has two objectives [8]. One is to ensure that for certain categories of motor vehicle noise limits of the different member states is not a barrier to trade, and the other one is to minimize the noise limits in order to reduce ambient noise. Although Directive 70/157/EEC managed to harmonize the procedure under test conditions and noise limits, it failed to reduce noise levels in real traffic, especially for cars. Real traffic conditions are other than testing conditions because tire noise has increased compared with powertrain noise and volume of traffic has increased continuously and will continue to grow. Because of this, the traffic noise was also discussed in EC Directive 42 (2001) and in the EC Regulation 661 (2009), relating to the rolling noise of tires and Directive 2002/49/EC concerning the assessment of environmental noise [4, 9-11].

Directive 2002/49/EC is implemented into the Romanian law by GD 674 (2007). This decision sets the general framework for the development of measures for reducing noise emitted by the major sources of noise, especially road vehicles, rail and their infrastructure, aircraft, industrial equipment sector, equipment for outdoors use and movable industrial machinery [12]. 


\section{ASSESSMENT OF ROAD TRAFFIC NOISE}

To achieve more accurate assessment is necessary to know the physical characteristics of the analysed areas. These features address the following issues:

- Geometrical configuration of the road infrastructure and topography of the analysed area refers to: roads in level, ramps, slopes, bridges, parking lots, multiple lanes roads, road sections in alignment curves, multiple perpendicular intersections, roundabouts etc.;

- Influence of the pavement of the tread on the acoustic emission levels of vehicles given by the surfaces coated with special absorbing materials, smooth asphalt, and other pavements like cubic stone, macadam etc.;

- Influence of the acoustic reflections caused by the existence of the buildings on the opposite side of the road section (canyon effect);

- Background noise level.

\subsection{Noise assessment through noise measurements}

Noise is characterized by physical parameters like: intensity, acoustic pressure, frequency, reverberation time. These parameters can be determined through noise measurements [13]. The aim of measuring the noise level generated by road traffic is to know its parameters and taking some adequate measures in order to reduce it where necessary. For the measurement and interpretation of data on noise levels generated by different sources and in different environments some recording equipment and software for data processing are necessary [13].

Noise measurements can be affected by atmospheric conditions and this influence may take place in different ways. First, the noise source itself may be affected - for example traffic has different characteristics when the road surface is wet. Secondly, the measurement equipment itself can be affected - for example, strong wind can cause noise in the microphone etc. At the same time, wind and rain can be powerful sources of noise themselves because of their actions on trees, buildings or road surfaces. As a result, atmospheric conditions require significant restrictions on the moments (periods) that measurements with an appropriate degree of reliability can be performed.

\subsection{Noise mapping}

Mapping is commonly used for the assessment of the noise. Regardless of the noise source, this procedure provides accurate information needed later in developing noise action plans. Mapping can be done through noise measurements or through calculation in the analysed areas and by using various specialized software $[14,15]$.

A noise map is a graphical representation of the noise level distribution in a certain area. Noise maps are drawn for two main purposes. First is to identify the areas where the noise level is high and where it is appropriate, and to calculate the number of people affected. This last point leads to the second and most important use of the noise maps, which is to be the basis of creating action plans to manage noise and reduce its harmful effects.

There are some technical and practical reasons for which noise maps are first generated using prediction/computer simulation than by direct measurement of noise. Thus, developing noise maps based on measurements will require many measurements that will be carried out over long periods of time and this will lead to very high costs. In most cases, in a location, the noise is a combination of local sources such as, for example, a combination of noise from road and rail. Normally, measurements cannot distinguish the contribution of each existing sources at the time of measurement, so, the problem can be solved by using computers to predict the noise levels for each individual noise source.

\section{METHODS FOR ASSESSING ROAD TRAFFIC NOISE}

Following the implementation of the European Directive on environmental noise, there have been used several methods to determine road noise. In Europe there have been used nine methods, of which the most popular being the French NMPB method. The methods NORD 1996 and NORD 2000 are commonly used by the Scandinavian countries, while Dutch RMW method is applied in countries such as Belgium and the Netherlands, Ireland uses the British CoRTN, while the rest of the member states have their own calculation methods or are using combinations of those listed. Figure 1 shows the regional distribution of different calculation methods used by the member states of the European Union for noise mapping [16]. 


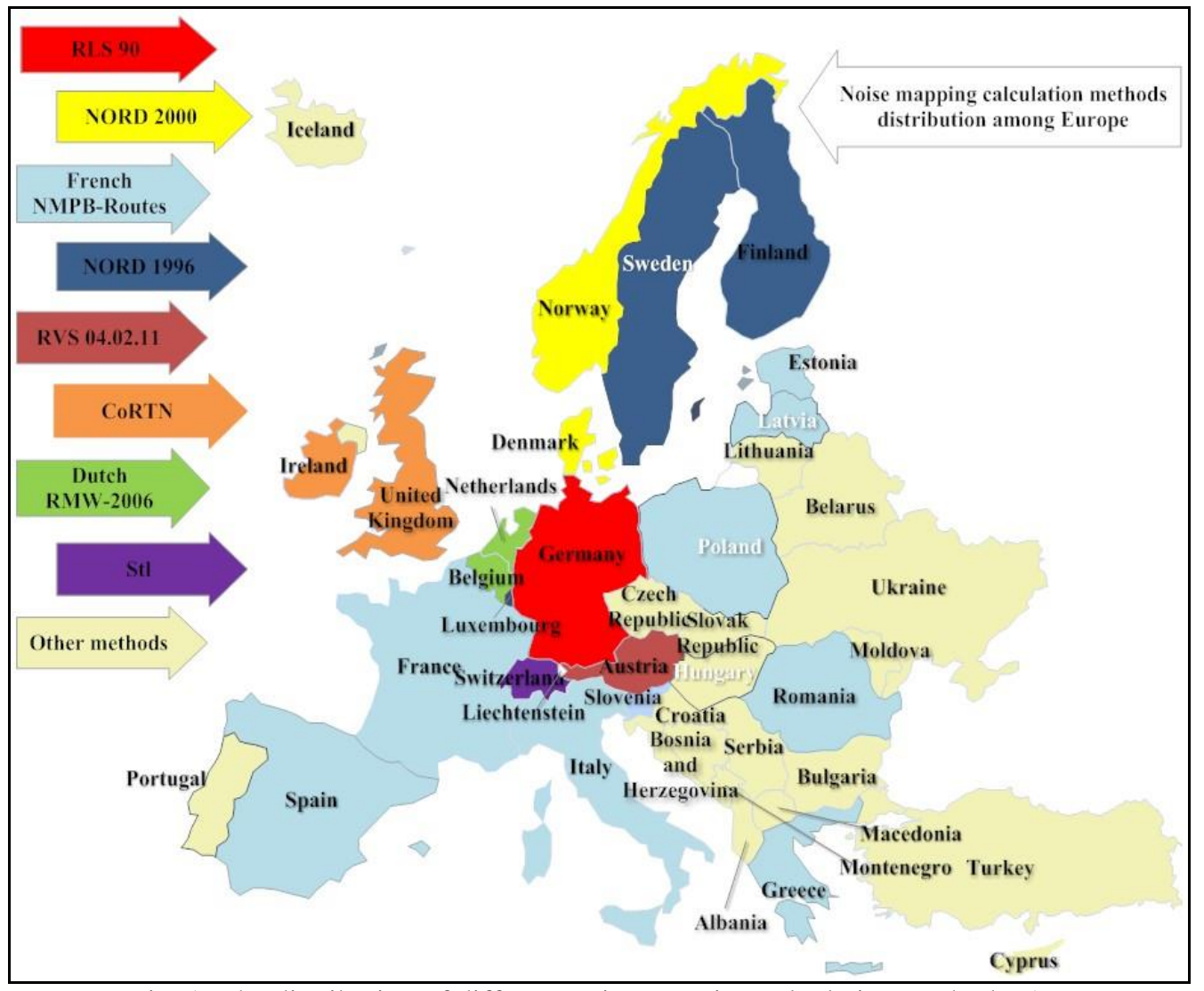

Fig. 1. The distribution of different noise mapping calculation methods [16]

For the member states which do not have national methods for calculating road noise or for the ones that wish to change their methods it is recommended to use interim computation methods for the determination of the common indicators $\mathrm{L}_{\mathrm{den}}$ and $\mathrm{L}_{\text {night }}[4]$.

\subsection{The method of noise propagation calculation NMPB 2008}

NMPB Method 2008 is the calculation method dealing with the propagation of sound emissions and it is intended for those departments responsible for the noise study in order to develop new projects of new residential areas or to correct the existing ones with high noise issues. The 1996 method version was used as the reference method in the European Noise Directive on the assessment and management of environmental noise. NMPB 2008 is one of the most widely used prediction method, being incorporated into many software used to calculate noise because it calculates noise level generated by a facility on a long-range taking into account weather conditions by analysing data collected on a long period of time $[4,6,17,18]$.

The calculation parameters, characteristic to the method, are [19]:

- $\quad$ Calculation per octave from $125 \mathrm{~Hz}$ to $4000 \mathrm{~Hz}$;

- 2-way propagation paths, favourable and neutral;

- Calculation of the percentage of favourable and neutral conditions based on the weather information, depending on wind direction angle of $20^{\circ}$;

- $\quad$ Characteristics of the rolling track (porous surface - grass infield).

The method is based on the noise propagation path principle, and it involves several paths between a source and a receiver, depending on topography and obstacles $[6,17-18,20]$.

The same method has been used in the prediction of road noise, for noise mapping purpose, in different urban areas from Romania, such as: Bucharest, Iasi, Timisoara, Brasov, Cluj-Napoca, Craiova etc. [21]. 


\subsection{Calculation of road traffic noise using the method CoRTN (UK)}

The method used mainly in the UK and Ireland was developed in 1975 by the Research Laboratory of Road and Transport, and the Department of Transport in the UK [18]. The parameters considered in this method are: traffic flow, the category of the vehicles participating in traffic, medium running speed, road gradient and type of the rolling track surface. Basic assumptions of the prediction model are calculated for a medium speed of wind and a dry runway $[2,17-18]$.

The CoRTN model involves five steps:

1. Dividing the road section in one or more segments such that the variation of the noise level in segment does not exceed $2 \mathrm{~dB}(\mathrm{~A})$;

2. Noise level calculation for each segment. Noise level depends on speed, traffic flow and on the category of the vehicles participating to the traffic. Traffic is considered a linear noise source placed at $0.5 \mathrm{~m}$ above the road surface;

3. Noise assessment for each segment, taking into account the attenuation due to distance and shielding of the linear source;

4. Estimation of noise considering reflected waves due to buildings and facades on the other side of the road and the size of the analysed segment;

5. Summing the noise levels of all segments to provide a noise prediction at the receiver all the way.

CoRTN model calculation formula is given by (equation 2) [2, 17-18]:

$$
L_{A 10(1 h)}=42.2+10 \log _{(q)}
$$

where $q$ is the hourly traffic flow calculated at the reference distance of $10 \mathrm{~m}$ from the nearest edge of the road and medium speed of $75 \mathrm{~km} / \mathrm{h}$;

\subsection{The RLS 90 prediction model (Richtlinien für den Lärmschutz an Straßen)}

This model was defined as an improvement of a previous standard RLS 81 and is the most used method in Germany which presents itself as an effective model of calculation to assess the level of road noise. The method requires the existence of input data regarding the hourly traffic flow average, separately for motorcycles, heavy and light vehicles, the medium speed for each group, size, geometry and type of the road and the obstacles of any kind, natural or artificial. Other factors taken into consideration are connected to the main characteristics influencing the propagation of noise, such as plants, absorption from the air, reflection and diffraction. Also, this method can make determinations on reducing road noise when noise barriers are used [17-18].

Studies have confirmed that the use of RLS 90 model has been properly in noise predicting and mapping. For example, in a study conducted in Chile, the method had accurate results regarding the prediction of road noise from the city of Santiago (Suárez and Barros, 2014). The values obtained in this study showed that noise levels in the city of Santiago, are high over a large area. These data were useful to the authorities to implement appropriate measures to minimize and remove potential sources of noise in the sensitive areas (residential areas, hospitals, schools) [22].

\subsection{Italian method of calculating noise C.N.R.}

This model is a modification of RLS 90 German standard, adapted to the Italian framework. The prediction method assumes the existence of a relationship between traffic parameters, the acoustic energy level and traffic flow, which is considered a linear noise source placed on the centre of the road [18]. Equivalent sound level for the noise prediction model is given by (equation 3 ):

$$
L_{a e q}=\alpha+10 \log \left(Q_{L}+\beta \cdot Q_{P}\right)-10 \log \left(d / d_{0}\right)+\Delta L_{V}+\Delta L_{F}+\Delta L_{B}+\Delta L_{S}+\Delta L_{G}+\Delta L_{V B}
$$

where $Q_{L}$ and $Q_{P}$ are the traffic flow in one hour reported to light and heavy vehicles; $d_{0}$ - reference distance of $25 \mathrm{~m} ; d$ - distance between the center line of the roadway and receiver; $\Delta L_{V}$ - noise variation due to the mean velocity; it is defined in Table $1 ; \Delta L_{F}$ and $\Delta L_{B}$ - variations given by the existence of reflective facade near the receiver $(2.5 \mathrm{~dB}(\mathrm{~A}))$, or in the opposite direction $(+1.5 \mathrm{~dB}(\mathrm{~A})) ; \Delta L_{S}$ - is the variation concerning the running surface; it is defined by the values given in Table $1 ; \Delta L_{G}$ - is the variation concerning the gradient of the road (the road angle) with a value greater than $5 \%$. The variation is $0.6 \mathrm{~dB}(\mathrm{~A})$ for each percentage greater than $5 \% ; \Delta L_{V B}$ coefficient which takes into account the presence of traffic lights $(+1.0 \mathrm{~dB}(\mathrm{~A}))$ or slow traffic $(-1.5 \mathrm{~dB}(\mathrm{~A}))$. 
All identified parameters have general validity, independent of other countries, however, the parameters $\alpha$ and $\beta$ are influenced by the characteristics of the roads from each country and by the vehicles which travel on these roads. The parameter $\alpha$ refers to noise generated by regular vehicles, and the parameter $\beta$ it is the weighting factor which takes into account the higher noise emission generated by heavy vehicles. Frequently, for Italian roads, the values of these parameters are assumed to be: $\alpha=35.1 \mathrm{~dB}(\mathrm{~A})$ and $\beta=6$ [18].

Table 1. The variation of noise level depending on vehicle speed and road surface [18]

\begin{tabular}{|c|c|c|c|}
\hline Flux mean speed $(\mathrm{km} / \mathrm{h})$ & $\Delta \mathrm{L}_{\mathrm{V}}(\mathrm{dB}(\mathrm{A}))$ & Road's pavement & $\Delta \mathrm{L}_{\mathrm{S}}(\mathrm{dB}(\mathrm{A}))$ \\
\hline $30-50$ & +0 & Smooth asphalt & -0.5 \\
\hline 60 & +1 & Rough asphalt & 0 \\
\hline 70 & +2 & Cement & +1.5 \\
\hline 80 & +3 & Rough pavement & +4 \\
\hline 100 & +4 & \multicolumn{2}{|}{} \\
\hline
\end{tabular}

\subsection{The FHWA noise prediction model, TNM (Federal Highway Administration)}

This noise prediction model is used in the United States. In March 1998, U.S. Federal Highway Administration launched the FHWA TNM road noise prediction to support policies and procedures in accordance with FHWA. This model is used to predict the noise impact in the vicinity of highways. By using advanced software it can be ensured data accuracy and ease highway noise modelling, including the construction of noise barriers, which are efficient in terms of cost $[17,23,24]$.

FHWA model uses as input data parameters related to: traffic flow, the distance between road and receiver, and adjusting the screens and ground effects between the road and the receiver. The calculation formula is given by (equation 4):

$$
L_{\text {Aeq, }, 1 h}=E_{L i}+A_{\text {traffic }(i)}+A_{d}+A_{s}
$$

where $A_{\text {traffic }}$ is the adjustment of traffic flow; $A_{d^{-}}$is the adjustment of the distance between road and receiver; $A_{s^{-}}$ is the adjustment of all screens and ground effects between road and receiver.

\subsection{Noise calculation method Harmonoise/IMAGINE}

Harmonoise/IMAGINE model is the conclusion of two consecutive European projects that tried to establish a common method of prediction of noise.

It was used by the member states of the European Union to develop accurate and valid strategic noise maps. The model defines the main categories of vehicles analysed as follows: light motor vehicles (category 1), medium (category 2), heavy vehicles (category 3) and motorcycles (category 4). Each category of vehicles is represented by two point sources, each having a specific sound power coming from the contact of the tire with the tread and from the propulsion noise [17].

The noise from rolling, $L_{W R}$ and the propulsion, $L_{W P}$ is given by the following equations:

$$
\begin{gathered}
L_{W R}(f)=a_{R}(f)+b_{R}(f) \log \left(v / v_{r e f}\right) \\
L_{W P}(f)=a_{P}(f)+b_{P}(f) \log \left[\left(v-v_{r e f}\right) / v_{r e f}\right]
\end{gathered}
$$

where $v_{r e f}-70 \mathrm{~km} / \mathrm{h} ; a_{r}, b_{r}, a_{p}, b_{p-} 1 / 3$ octave band coefficient for a domain of frequency between $25 \mathrm{~Hz}$ and $10 \mathrm{kHz}$.

In assessing the road traffic noise, suitable prediction methods have an important role because they allow the assessment of the environmental impact for existing infrastructure and there are also useful in the design of new road networks so that noise is minimized where it is necessary [25]. Harmonoise/IMAGINE overcome some problems detected in the rest of the national models regarding the estimation of the emitted noise power of the road itself. For example, the issues related to the acceleration/deceleration can be calculated in an accurate way. 
Another example is about the real height of the linear sources (propulsion and rolling noise) which the model takes into account.

\subsection{Common Noise Assessment Method in Europe CNOSSOS}

In 2009 the European Commission decided to develop Knossos-EU (Common Noise Assessment Methods in Europe) for noise mapping of road, rail, aviation and industry in order to develop a methodological framework for the evaluation of noise $[19,25]$. CNOSSOS-EU method applies to the determination of noise frequency of 12 $\mathrm{Hz}$ to $4 \mathrm{kHz}$ for road traffic noise. Vehicles are divided into four separate categories on noise emission characterization, while the fifth category is foreseen as an open category for vehicles that can be developed in the future [17]. For each vehicle, the transmission pattern consists of a set of mathematical equations representing the two main sources of noise such as: rolling noise and the propulsion noise. The coefficients are given in octave bands for each category of vehicle for a reference speed equal to $70 \mathrm{~km} / \mathrm{h}$ and virtual reference road surface consisting of an average of dense asphalt concrete stone asphalt. For areas with other features, the recommendation is to apply a spectral correction factor regarding the rolling noise and in case of a porous surface to apply correction factors on both main sources of noise. Equivalent continuous sound pressure level at a receiver is calculated corresponding to the two types of atmospheric conditions, namely: downward refraction and homogeneous atmospheric conditions [17].

In assessing road traffic noise suitable prediction methods have an important role because they allow the environmental impact assessment in the case of existing infrastructure and are also useful in the design of road networks so that noise is minimized where the infrastructure will be built [25].

From the noise prediction methods presented in this summary, the method NMPB 2008 is the most commonly used in forecasting urban traffic noise, being recommended as the reference method in European Directive 2002/49/EC on the assessment and management of environmental noise. European member states that do not have a method of self-assessment, and states that wish to change their method of calculating the urban road noise, can use this method. Other states have their own noise prediction methods or they combine a number of features from several methods. These assessments are required for mapping the urban road noise and elaborating the future action plans to reduce noise where necessary.

\section{CONCLUSIONS}

Noise mapping requires both knowledge of the analysed area (geographical approach), and the attributes that define the noise source, the propagation path and the receivers. All the models use this information in order to define the sound power of the source and the propagation model.

The prediction models replace efficiently the measurements on site. It is well known that the prediction models incorporated in the software packages show a lot of advantages compared to the development of noise maps through the use of noise measurements.

The noise source represented by traffic is determined by summing the traffic noise emissions generated by each vehicle in the traffic flow. Some of the problems arise when the noise emitted by these vehicles is not geometrically properly defined. Harmonoise considers different heights for the emission of the different categories of vehicles but for example NMPB considers only one height. This has a direct consequence on the propagation and, hence on the effectiveness of several noise mitigation measures (noise barriers).

There are many other factors that are not well taken into account in the noise prediction models. This leads to a not very precise estimation of road traffic noise. The analysed models do not consider enough categories of vehicles and road surfaces, age of the vehicles, the major part do not include a good approach to stop and go behaviour of drivers and the influence of the slopes. Another important aspect is the poor capacity of the major part of the models in making an estimation of noise for vehicles running under $30 \mathrm{~km} / \mathrm{h}$, which is a regular speed in urban areas.

Regular assessment of road traffic noise is required to maintain under control noise levels in busy urban areas or to explore the possibility of developing new projects of residential constructions or other buildings like: schools, hospitals etc. on certain busy roads, where traffic noise can be a problem. It is necessary to understand the pros 
and cons and the limitation of the models in order to choose the best one for complying the requirements of the noise mapping project.

\section{REFERENCES}

[1] Arvind, K.S., Sukhvir, S.J., Parida, M., Srivastava, J.B., Performance of traffic noise models for metropolitan cities, Environmental Engineering and Management Journal, vol. 7, p. 447-452, 2008.

[2] Kelly, D., Traffic noise impact assessment Dublin waste to energy facility, Technical Report, AWN Consulting, kellyDublin, 2006, online at: http://dublinwastetoenergy.ie/uploads/Uploads/Traffic\%20Noise\% 20Impact\%20Assessment.pdf.

[3] LK NOISE SRL, SNM - Strategic noise mapping - a tool for global assessment of noise exposure, 2012 , (Romanian version) online at: http://50.6.191.177/node/4.

[4] EC Directive 49, relating to the assessment and management of environmental noise (the Environmental Noise Directive - END), 2002.

[5] Lipscomb, D.M., Taylor, A.C., Noise Control: Handbook of Principles and Practices, Van Nostrand Reinhold Co, 1978.

[6] Dutilleux, G., Defrance, J., Gauvreau, B., Besnard, F., The revision of the French method for road traffic noise prediction, The Journal of the Acoustical Society of America, vol. 123, p. 875-880, 2008.

[7] EEA, Noise. Environmental noise affects a large number of Europeans, European Environment Agency, 2011, online at: http://www.eea.europa.eu/downloads/049ee3266f000c305070cf21fd3503fa/1339163726/ intro.pdf.

[8] EC Directive 157, (1970), Council Directive 70/157/EEC of 6 February 1970 on the approximation of the laws of the Member States relating to the permissible sound level and the exhaust system of motor vehicles, Official Journal of the European Communities, L42, p. 111-116, 1970.

[9] CSWD, Summary of the impact assessment which accompanies the document: Proposal for a Regulation on the sound level of motor vehicles, Comission Staff Working Document, Bruxelles, 2011, online at: http://www.europarl.europa.eu/RegData/docs_autres_institutions/commission_europeenne/sec/2011/1504/COM _SEC(2011)1504_RO.pdf.

[10] EC Directive 43, of the European Parliament and of the Council of 27 June 2001 amending Council Directive 92/23/EEC relating to tires for motor vehicles and their trailers and to their fitting, 2001.

[11] EC Regulation 661, regulation (EC) No 661/2009 of the European Parliament and of the Council of 13 July 2009 concerning type-approval requirements for the general safety of motor vehicles, their trailers and systems, components and separate technical units intended therefor, Official Journal of the European Communities, L200, p. 1-24, 2009.

[12] GD 647, Governmental Decision No. 674 from 28 of June, (2007), for amending and supplementing Government Decision no. 321/2005 on the assessment and management of environmental noise, Romanian Official Monitor, no. 485, p. 1-24, 2007, online at: http://www.romanian-ports.ro/legimediu/HG674_2007.pdf.

[13] Tomozei, C., Nedeff, V., Panainte, M., Lazăr, G., Nedeff, F., Modalitati de masurare a zgomotului aerian, in Reducerea poluarii fonice in mediul industrial, Alma Mater publishing house, Bacau, Romania, p. 123 -149, 2014

[14] Den Boer, L.C., Schroten, A., Traffic noise reduction in Europe: Health effects, social costs and technical and policy options to reduce road and rail traffic noise, Report, Delft, the Netherlands, 27-282008, online at: http://www.transportenvironment.org/sites/te/files/media/2008-02 traffic noise ce delft report.pdf.

[15] Platon, S. N., Hionis, C. A., Preventing risk of noise exposure in working environment using noise mapping, Environmental Engineering and Management Journal, vol. 13, p. 1349-1354, 2014.

[16] BPSNP, Best practice in noise strategic mapping, CEDR, 2013, online at: http://www.fomento.gob.es/NR/rdonlyres/CB0F3D92-E801-47AD-9028-

FC7E306D3E97/120805/1CEDRJRNoiseMapping_August_2013.pdf.

[17] Garg, N., Maji, S., A critical review of principal traffic noise models: Strategies and implications, Journal of Environmental Impact Assessment Review, vol. 46, p. 68-81, 2014.

[18] Quartieri, J., Mastorakis, N. E., Iannone, G., Guarnaccia, C., D’Ambrosio, S., Troisi, A., Lenza, TLL., A Review of Traffic Noise Predictive Models, Proc. of the 5th WSEAS International Conference on Applied and Theoretical Mechanics, December 14-16, Puerto De La Cruz, Tenerife, Canary Islands, Spain, p. 72-80, 2009.

[19] EC, CNOSSOS - Common Noise Assessment Methods in Europe, 2012, online at: http://www.envi.ro/index.php?view=news\&action=viewnews\&newsid=225.

[20] Mercha Iglesias, C., Balteiro Diaz, L., Noise pollution mapping approach and accuracy on landscape scales, Science of the Total Environment, vol. 449, p. 115-125, 2013. 
[21] ANPM, Noise Strategic Maps for different agglomerations in Romania, 2009, online at: http://www.anpm.ro/files2/10\%20MEDIUL\%20URBAN\%20ANEXE_200910161128234.pdf.

[22] Suárez, E., Barros, J.L., Traffic noise mapping of the city of Santiago de Chile, Science of the Total Environment, vol. 466-467, p. 539-546, 2014.

[23] Kumar, K., Parida, M., Katiyar, V. K., Prediction of urban traffic noise using artificial neural network approach, Environmental Engineering and Management Journal, vol. 13, p. 817-826, 2014.

[24] Volpe, J.A., FHWA Traffic Noise Modeluser's Guide (version 2.5 addendum), 2004, online at: http://www.fhwa.dot.gov/environment/noise/traffic noise model/tnm_v25/users manual/TNM25UsersGuideAd dendum.pdf.

[25] Kephalopoulos S., Paviotti M., Anfosso-Lédée F., (2012), Noise Assessment Methods in Europe (CNOSSOS-EU), online at: http://publications.jrc.ec.europa.eu/repository/bitstream/111111111/26390/1/ cnossos-eu\%20jrc\%20reference\%20report_final_on\%20line\%20version_10\%20august\%202012.pdf. 